\title{
Cardiac corticosteroid receptors mediate the enlargement of the ovine fetal heart induced by chronic increases in maternal cortisol
}

\author{
Seth A Reini ${ }^{1}$, Garima Dutta, Charles E Wood ${ }^{\mathbf{1}}$ and Maureen Keller-Wood \\ Department of Pharmacodynamics, College of Pharmacy, University of Florida, Box 100487, Gainesville, Florida 32610-0487, USA \\ ${ }^{1}$ Department of Physiology and Functional Genomics, College of Medicine, University of Florida, Gainesville, Florida 32610-0274, USA \\ (Correspondence should be addressed to M Keller-Wood; Email: kellerwd@cop.ufl.edu)
}

\begin{abstract}
Previous studies have demonstrated that modest, physiologically relevant increases in maternal cortisol in late gestation result in enlargement of the fetal heart. In this study, we investigated the role of mineralocorticoid receptor (MR) or glucocorticoid receptor (GR) in this enlargement. Ewes with single fetuses were randomly assigned at $\sim 120$ days of gestation to one of four groups: maternal cortisol infusion (1 mg/kg per day, cortisol); maternal cortisol infusion with fetal intrapericardial infusion of the MR antagonist $(\mathrm{MRa})$ potassium canrenoate $(600 \mu \mathrm{g} /$ day; cortisol+MRa); maternal cortisol infusion with fetal intrapericardial infusion of the GR antagonist (GRa) mifepristone $(50 \mu \mathrm{g} / \mathrm{day}$, cortisol+GRa); and maternal saline infusion (control). At $\sim 130$ days of gestation, fetal heart to body weight ratio and right ventricular (RV) and left ventricular (LV) free wall thicknesses were increased in the cortisol group
\end{abstract}

when compared with control group. Fetal hearts from the cortisol $+\mathrm{MR}$ a group weighed significantly less, with thinner $\mathrm{LV}, \mathrm{RV}$, and interventricular septum walls, when compared with the cortisol group. Fetal hearts from the cortisol+GRa group had significantly thinner RV walls than the cortisol group. Fetal arterial pressure and heart rate were not different among groups at 130 days. Picrosirius red staining of fetal hearts indicated that the increased size was not accompanied by cardiac fibrosis. These results suggest that physiologic increases in maternal cortisol in late gestation induce fetal cardiac enlargement via MR and, to a lesser extent, by GR, and indicate that the enlargement is not secondary to an increase in fetal blood pressure or an increase in fibrosis within the fetal heart.

Journal of Endocrinology (2008) 198, 419-427

\section{Introduction}

In late gestation, normal fetal growth and fetal cardiovascular homeostasis is dependent on the proper regulation of maternal cortisol levels. Although reductions in maternal cortisol prevent the normal increases in maternal plasma volume and uteroplacental blood flow and reduce fetal growth (Jensen et al. 2002a, 2005), increases in maternal cortisol also alter fetal growth. Chronically increased maternal cortisol levels, within the range that occurs with maternal stress, reduce fetal growth rates while increasing heart growth in fetal sheep (Jensen et al. 2002b, 2005).

The mechanisms by which chronically elevated maternal cortisol levels increase the size of the fetal heart are not known. Giraud et al. (2006) have shown that cortisol chronically infused directly into the coronary artery increased cell cycle activity in myocytes of late gestation sheep fetuses, suggesting a direct induction by cortisol of hyperplastic growth rather than hypertrophic growth. Conversely, it has been demonstrated that large doses of cortisol infused directly into the fetus in late gestation causes left ventricular (LV) hypertrophy along with an increase in fetal arterial pressure and cardiac expression of angiotensinogen mRNA (Lumbers et al. 2005). We have shown that maternal cortisol infusion in sheep during late gestation caused an increase in fetal heart size and wall thickness without increasing fetal arterial pressure or cardiac angiotensinogen; we found an increase in the ratio of angiotensin type 2 receptor (AT2 receptor) to AT1 receptor mRNA in the fetal heart, suggesting that the renin-angiotensin system (RAS) may play a key role in the enlargement process. Furthermore, in the same study it was observed that LV expression of $11 \beta$-hydroxysteroid dehydrogenase (11 $\beta$-HSD2) mRNA, the enzyme that converts cortisol into cortisone, decreased in the fetal hearts in response to the elevated cortisol, suggesting that cortisol can act directly on mineralocorticoid receptors (MRs) or glucocorticoid receptors (GRs) to induce the cardiac enlargement (Reini et al. 2006).

In adult hearts, both the MR and the RAS have been implicated in cardiac fibrosis and hypertrophy after injury (Fraccarollo et al. 2003, 2005, Xiao et al. 2004). We propose that corticosteroid receptors also play a role in cardiac enlargement in the fetal heart, although by mechanisms independent of cardiac injury and fibrosis. The purpose of this 
study was to test the hypothesis that increase in fetal heart weight and wall thickness in response to increased maternal cortisol is mediated by cardiac corticosteroid receptors, MR and/or GR, and to determine if cardiac fibrosis accompanies the cardiac enlargement in response to cortisol. We hypothesized that cortisol acts within the myocardium on MRs, and to a lesser degree on GRs, to induce enlargement of the fetal heart. We also hypothesized that cardiac fibrosis is not involved in the enlargement of the heart observed in the fetuses of cortisol-infused ewes.

\section{Materials and Methods}

\section{Experimental design}

Ewes (Ovis aries) pregnant with single fetuses were studied. All animal use was approved by the University of Florida Institutional Animal Care and Use Committee and conformed to the National Institutes of Health Guide for the Care and Use of Laboratory Animals. Ewes and their fetuses were operated on between 118 and 123 days of gestation (term 148 days). Animals were randomly assigned to one of four groups at the time of surgery. The first group consisted of six control ewes infused with saline; the second group consisted of five ewes to which cortisol (hydrocortisone hemisuccinate; Sigma) was administered by continuous i.v. infusion (1 mg/kg per day; cortisol); the third group consisted of six ewes to which cortisol was infused, with infusion of the MR antagonist potassium canrenoate (Sigma; $600 \mu \mathrm{g} /$ day; cortisol+MRa) directly into the pericardial space of the fetus; and the fourth group consisted of four ewes to which cortisol was infused, with infusion of the GR antagonist mifepristone (Sigma; $50 \mu \mathrm{g} /$ day; cortisol + GRa) directly into the pericardial space of the fetus. For the control and cortisol groups, there were no infusions into the pericardial space. The intrapericardial infusions were performed by use of Alzet minipumps (model 2ML2; $5 \mu \mathrm{l} / \mathrm{h}$; Durect Corporation, Cupertino, CA, USA) in order to achieve continuous infusion of the antagonists into the pericardial space without any appreciable increase in pericardial fluid volume $(0.12 \mathrm{ml} /$ day $)$. The doses of MR and GR antagonists were calculated based on their effective systemic doses, and scaled to reflect the smaller distribution volume of the fetal heart $(20 \mathrm{~g})$. Because these drugs are steroid (mifepristone) or lactone (canrenoate) derivatives, they are able to distribute throughout the tissue over the 10 days of study after mixing in the pericardial fluid. Effects of the MR and GR antagonists were confirmed using immunohistochemistry to confirm the expected cellular redistribution of receptors with antagonist administration (see below).

The cortisol dose and the duration of cortisol infusion (10 days) were determined based on a previous study in this laboratory (Jensen et al. 2005) showing that infusion at this rate and duration produces levels similar to mild maternal stressors and results in enlargement of the fetal heart.

\section{Surgical procedures}

Halothane $(1 \cdot 5-2 \cdot 5 \%)$ in oxygen was used to anesthetize ewes during surgery. Fetal femoral tibial artery catheters and an amniotic fluid catheter were placed as described previously (Wood \& Rudolph 1983, Jensen et al. 2002a). Catheters were also placed in the fetal pericardial space for the delivery of the drug as described previously (Wood 2002). In each case, an incision was made in the uterus over the left side of the fetal chest and an incision was made between the third and fourth fetal ribs. The fetal skin was marsupialized to the uterus to prevent leakage of amniotic fluid. The fetal heart was exposed and a small incision was made in the pericardium, through which a silastic catheter (0.76 mm i.d., $1.65 \mathrm{~mm}$ o.d.; Dow Corning, Midland, MI, USA) was placed and held in place with the use of a pursestring suture (4-0 Tevdek; Teleflex Medical, Mansfield, MA, USA). For infusion of potassium canrenoate, the silastic catheter was connected to a Tygon tubing connector $(1.27 \mathrm{~mm}$ o.d.; St Gobain Performance plastics; Akron, OH, USA) which was connected at its other end to the Alzet pump containing the drug $(50 \mathrm{mg} / \mathrm{ml}$ in $0.9 \%$ saline). Because mifepristone is not soluble in aqueous solution and therefore cannot be directly loaded into the pump reservoir, mifepristone was dissolved in $47.5 \%$ ethanol in saline $(0.42 \mathrm{mg} / \mathrm{ml}$ ethanol-saline $)$; this solution was placed in a polyethylene tubing $(1.40 \mathrm{~mm}$ i.d., $1.90 \mathrm{~mm}$ o.d.) which was then connected to the silastic pericardial catheter on one end and to the Alzet minipump on the other end using smaller gauge polyethylene tubing. The Alzet pump, filled with saline, provided the flow to pump the mifepristone solution from the tubing into the pericardium. The pump was placed under the skin of the fetus near the scapula. In the control group, five of six fetuses also had pericardial catheters placed, but no infusion was delivered; in the cortisol group, three of five fetuses had pericardial catheters placed, but no infusion was delivered.

After closure of the uterus, catheters were placed in the maternal femoral artery and vein and routed to the maternal flank. All ewes were treated with flunixamine $(1 \mathrm{mg} / \mathrm{kg}$ i.m.; Fort Dodge Animal Health, Fort Dodge, IA, USA) at the end of the surgical procedure, before recovery from anesthesia.

Ewes were returned to their pen after recovery from anesthesia. At this time, the i.v. infusion of cortisol $(1 \mathrm{mg} / \mathrm{kg}$ per day cortisol as cortisol hemisuccinate in normal saline; Sigma) or infusion of saline to the ewe was initiated. Maternal infusions were delivered through a $0.22 \mu \mathrm{m}$ filter (Millipore Corp. Medford, MA, USA) via a syringe pump at the rate of $1.17 \mathrm{ml} / \mathrm{h}$. Animals were housed in individual pens with access to water, food, and salt blocks ad libitum. Ampicillin (500 mg i.m. bid; Webster Veterinary) was administered for 3 days postoperatively. Flunixamine was administered on the morning after surgery.

\section{Experimental protocol}

Fetuses were studied from the day of surgery until death on 129-132 days of gestation. All cortisol-infused ewes and their 
fetuses were killed on day 10 of infusion. Fetal and maternal blood samples were withdrawn on day 5 (124-126 days of gestation) and day 10 (129-132 days of gestation) after the start of the infusion for determination of blood gases, plasma cortisol, and plasma adrenocorticotropin (ACTH) concentrations. All blood samples were taken immediately after entering the room in which the ewes were housed in order to minimize the effect of handling on plasma ACTH and cortisol. On day 10 of infusion, maternal and fetal blood pressure and heart rate were recorded over a 40-min interval using LabView software (National Instruments, Austin, TX, USA) and disposable pressure transducers (Transpac; Hospira, Lake Forest, IL, USA) Amniotic fluid pressures were subtracted from fetal intra-arterial pressures in order to calculate fetal arterial pressure. In two animals, one in the cortisol group and one in the cortisol + MRa group, we were unable to reliably measure fetal heart rate; data from those two fetuses are excluded from analysis.

The ewe was euthanized on day 10 using an overdose of euthanasia solution containing pentobarbital, and the fetus was removed and weighed. The fetal heart was also immediately dissected, blotted to remove blood from the chambers, and weighed. Ventricular and septal wall thicknesses were measured using a micrometer at a standardized site on the heart, taking care to exclude measurement at the level of the papillary muscles or valves.

\section{Analysis}

Blood gases and $\mathrm{pH}$ were measured with a blood gas/electrolyte analyzer (ABL77; Radiometer America, Westlake, OH, USA). Electrolytes (sodium and potassium) were measured using an electrolyte analyzer (Roche 9180). For measurement of packed cell volume (PCV), blood was spun in microcapillary tubes for $3 \mathrm{~min}$ at $5000 \mathrm{~g}$. (Damon Division, International Equipment, Needham Heights, MA, USA). Plasma protein was determined using a refractometer.

Plasma ACTH was measured by RIA, using an antibody to ACTH (1-39) (Bell et al. 1991), and plasma cortisol concentration was measured using a commercially available enzyme immunoassay kit (EA 65, Oxford Biomedical, Oxford, MI, USA) that has minimal cross-reactivity with cortisone $(2 \cdot 08 \%)$.

\section{Immunohistochemical localization of MR and GR}

At the time of killing, a section of the fetal heart was fixed in $4 \%$ buffered paraformaldehyde overnight. Hearts were dehydrated with increasing concentrations of reagent alcohol followed by xylene, embedded in paraffin wax, cut into $10 \mu \mathrm{m}$ thick sections on a Zeiss rotary microtome, and placed on poly-L-lysine coated slides. The sections were stained with anti-GR (Santa Cruz Biotechnology, Santa Cruz, CA, USA M-20) or anti-MR (M1-18, 6G1, gift of Gomez-Sanchez et al. (2006)) as described previously (Reini et al. 2006) This analysis was performed to assess the ability of the drugs to act in the heart and cause the expected changes in cytonuclear localization of the receptors. The MR antagonist potassium canrenoate acts in a similar manner to spironolactone and would therefore be expected to prevent nuclear localization of MR (Lombes et al. 1994, Fejes-Toth et al. 1998); conversely the GR antagonist mifepristone (also known as RU486) causes nuclear localization even in the absence of agonist (Jewell et al. 1995, Scheuer et al. 2004). The localization observed (Fig. 1) is consistent with these effects. In the control fetuses GRs were primarily located in the cytosol, whereas MR was apparent in the cytosol and nucleus. A dramatic increase in MR localization to the nucleus was apparent in the cortisol-treated fetuses, indicating cortisol activation of MR. We did not find as dramatic an increase in nuclear GR with cortisol, indicating that fewer GR are activated. In the case of MR antagonist administration, fewer MR were apparent in the nucleus than with cortisol alone, whereas with GR antagonist, equivalent MR localization to the nucleus occurred as with cortisol alone. Consistent with the known effect of mifepristone, in GR antagonist-treated ewes, there was more nuclear GR than in the case of cortisol alone or cortisol+MRa.

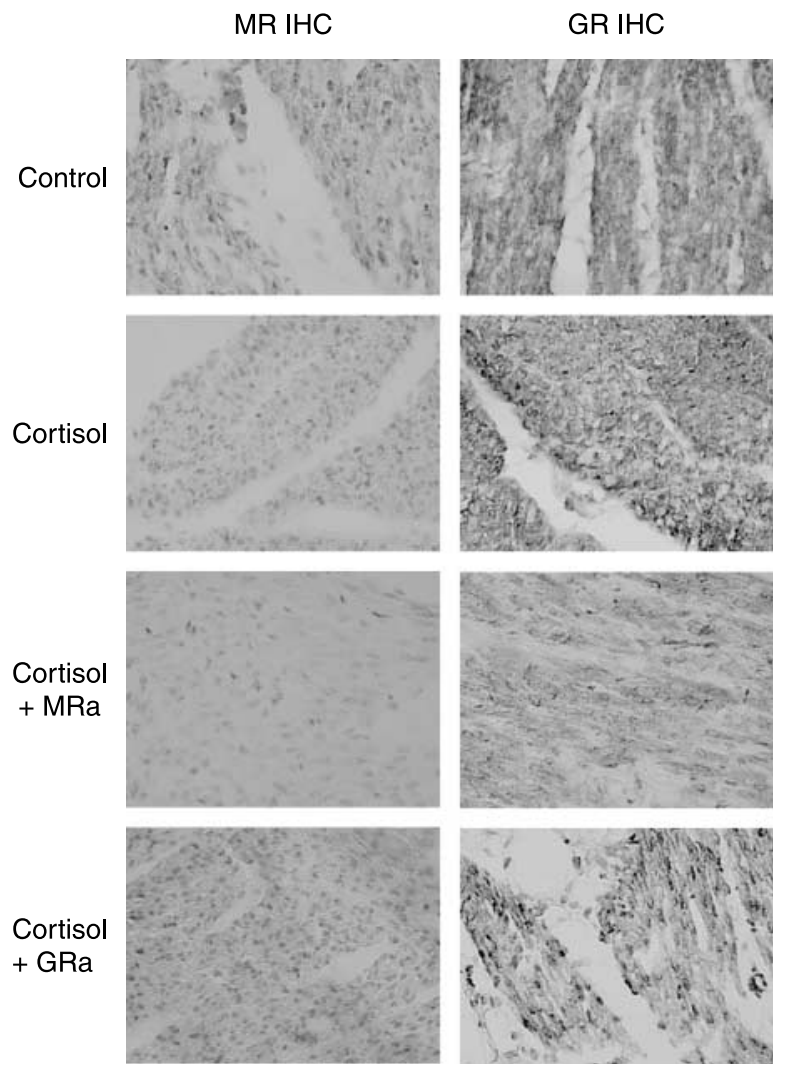

Figure 1 Immunohistochemical localization of MR (left column) and GR (right column) in representative hearts from fetuses of control, cortisol, cortisol + MRa, and cortisol + GRa groups. All photos at $40 \times$ power. 


\section{Collagen staining}

Sections from each group $(n=4-6)$ were stained with picrosirius red (Sigma) in order to determine collagen content. Sections were hydrated and immersed in Sirius red $(0 \cdot 1 \%$ in saturated picric acid). The sections were then washed in acidified water $(0 \cdot 5 \%$ glacial acetic acid), dehydrated, and mounted in permount. All images were visualized using an Olympus DP71 microscope and Olympus software. Ten pictures of LV, five of right ventricle (RV) and five of septum, were taken from each heart in areas without large blood vessels so that primarily interstitial, rather than perivascular, collagen deposition could be quantified. Picrosirius red staining was quantified using Image $\mathrm{J}$ software $(\mathrm{NIH}$, Bethesda, MD, USA) by three different people who were blinded as to the experimental group. The average value of the percentage of the image that stained red from these three observations was calculated.

\section{Data analysis}

Fetal heart weight was normalized to body weight. The heart weight to body weight ratio, LV, RV, and septal thickness, fetal and maternal blood pressure and heart rate, as well as fetal and maternal plasma ACTH and cortisol, sodium and potassium, and PCVs were analyzed by one-way ANOVA with multiple comparisons using Duncan's method (Zar 1984). Plasma hormone (cortisol and ACTH) and protein concentrations were also analyzed by one-tailed $t$-test, comparing the data from all three groups of cortisol-treated ewes to the data from the control group (Zar 1984). Average cortisol values were calculated from the day 5 and day 10 values and were log transformed before analysis. The Mann-Whitney rank sum test was used for maternal plasma protein analysis at day 10 (Zar 1984).

Values for the picrosirius red staining were analyzed by two-way ANOVA in order to determine significance across the cortisol treatment groups and areas of the heart $(\mathrm{LV}, \mathrm{RV}$, and septum); the percent stained area data were transformed using arcsine prior to analysis to correct for heteroscedascity (Winer 1971).

For all analyses, $P<0.05$ was used as the criterion for statistical significance.

\section{Results}

\section{Maternal physiology}

Maternal cortisol concentrations were significantly increased in the ewes treated with cortisol when compared with the non-treated ewes (average of days 5 and 10, 9.0 $\pm 0 \cdot 9$ vs $5 \cdot 9 \pm$ $1.4 \mathrm{ng} / \mathrm{ml}$ ). When the four groups were compared individually, there was a trend for each cortisol-treated group to have increased cortisol concentrations when compared with the control ewes (Table 1), but there were no differences among groups. ACTH levels were not significantly altered in response to cortisol treatment, although there was a trend for cortisol-treated ewes to have lower ACTH concentrations than the control group (Table 1).

Maternal sodium, potassium, and PCV values were not different between the groups at day 5 or day 10 (data not shown). Maternal plasma protein concentrations were significantly elevated in the cortisol-treated ewes on days 5 $(8 \cdot 2 \pm 0 \cdot 2$ vs $7 \cdot 6 \pm 0 \cdot 2 \mathrm{~g} 100 / \mathrm{ml})$ and $10(7 \cdot 9 \pm 0 \cdot 1$ vs $7 \cdot 4 \pm$ $0 \cdot 1 \mathrm{~g} 100 / \mathrm{ml}$ ) when compared with the control ewes.

Maternal arterial pressures and heart rates were not different between the four groups (data not shown).

\section{Fetal physiology}

The average plasma cortisol concentrations (days 5 and 10) were significantly elevated in the fetuses whose mothers were infused with cortisol compared with control $(3 \cdot 4 \pm 0 \cdot 6$ vs $1.5 \pm 0.6 \mathrm{ng} / \mathrm{ml})$. There was a trend for each cortisol-treated group to have increased cortisol concentrations when compared with the control fetuses when the four groups were compared individually (Table 1). ACTH levels were not significantly altered in response to cortisol treatment (Table 1).

There were no significant differences among the groups in the blood gas values or PCV (Table 2), nor were there effects on fetal electrolytes (data not shown). There were also no effects of treatment on fetal heart rate and blood pressures (Table 3).

\section{Fetal heart measurements}

Heart weight was significantly greater in the cortisol group when compared with the control group and cortisol $+\mathrm{MRa}$ group, but not the cortisol+ GRa group (Fig. 2). LV and RV free wall thicknesses were significantly greater in the fetal hearts of the cortisol-treated group when compared with the control group. LV and RV free wall, as well as septum thicknesses were greater in the fetal hearts of the cortisol group when compared with the cortisol+MRa group (Fig. 2). LV free wall and septum thicknesses were not different in the cortisol group when compared with the cortisol+GRa group (Fig. 2). However, RV free wall thickness was greater in the cortisol group when compared with the cortisol+ GRa group.

\section{Collagen staining}

Fetal heart sections were stained with picrosirius red in order to measure the amount of interstitial collagen deposition (Fig. 3). The percentage of collagen staining in the LV, RV, septum, and whole heart was not significantly altered among the groups (Table 4, Fig. 3). 
Table 1 Fetal and maternal cortisol concentrations (average of days 5 and 10) and ACTH concentration on day 10. Data are expressed as mean \pm S.E.M

\begin{tabular}{|c|c|c|c|c|}
\hline & Maternal cortisol $(\mathrm{ng} / \mathrm{ml})$ & Fetal cortisol (ng/ml) & Maternal ACTH (pg/ml) & Fetal ACTH $(\mathrm{pg} / \mathrm{ml})$ \\
\hline Control & $5 \cdot 9 \pm 1 \cdot 4$ & $1 \cdot 5 \pm 0 \cdot 6$ & $37 \pm 8$ & $36 \pm 8$ \\
\hline Cortisol & $9 \cdot 6 \pm 2 \cdot 3$ & $2 \cdot 7 \pm 0 \cdot 5$ & $20 \pm 1$ & $27 \pm 5$ \\
\hline Cortisol + MRa & $8 \cdot 7 \pm 0 \cdot 4$ & $3 \cdot 6 \pm 1 \cdot 0$ & $31 \pm 11$ & $38 \pm 6$ \\
\hline Cortisol + GRa & $8 \cdot 3 \pm 1 \cdot 5$ & $3 \cdot 9 \pm 1 \cdot 9$ & $21 \pm 1$ & $57 \pm 33$ \\
\hline
\end{tabular}

\section{Discussion}

We conclude that blockade of corticosteroid receptors in the fetal heart prevents the enlargement of the heart observed when maternal cortisol concentrations are chronically increased. We found that blockade of the MRs blocked the increase in heart weight, as well as wall thickness. Blockade of GRs significantly reduced RV enlargement, and produced smaller insignificant effects on thickness of the LV free wall and septum and on heart weight. Administration of neither MR nor GR blocker into the pericardium resulted in increases in fetal ACTH or fetal blood pressure, suggesting that the infusions of antagonist did not produce systemic effects. The results indicate that small increases in cortisol increase fetal heart size via an intracardiac action at the MRs and, to a lesser extent, GRs within the fetal heart. We also conclude that the increase in fetal heart weight in response to elevated cortisol occurs without an increase in collagen deposition (Fig. 3).

\section{Role of MR and GR in the heart}

Our laboratory has previously shown that both MR and GR are abundantly expressed in the heart in late gestation ovine fetus (Reini et al. 2006), suggesting a role for these receptors in fetal heart development in vivo. Other investigators have found that aldosterone directly stimulates myocyte surface area (Okoshi et al. 2004) and remodeling of myocyte membrane (Kliche et al. 2006) in cultures of neonatal myocytes, an effect presumed to be mediated by MR in the myocytes. Cortisol also increases expression of atrial natriuretic peptide in cultured neonatal myocytes, and both cortisol and aldosterone potentiate the effect of phenylephrine on hypertrophy in these cultures (Lister et al. 2006), also indicating an intracardiac action at MR in these cultures.
One of the major factors influencing the ability of cortisol to activate MR and/or GR is the local activity of the $11 \beta$ -

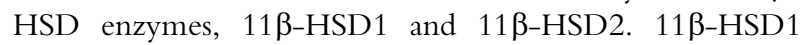
primarily converts cortisone into cortisol, while $11 \beta-H S D 2$ converts cortisol into cortisone, which is inactive at MR and GR (Krozowski et al. 1999). We had previously shown that mRNA expression of $11 \beta-H S D 2$ mRNA is relatively low compared with 11ß-HSD1 within the fetal heart (Reini et al. 2006). Using immunohistochemistry, we also found that although MR, GR, and 11 $\beta$-HSD1 are abundantly expressed in both myocytes and blood vessels within the fetal heart, $11 \beta-H S D 2$ seemed to be localized in blood vessels more abundantly than in myocytes. This suggested that cortisol has access to both MR and GR within the fetal heart, and that when plasma cortisol levels are increased, as in the present study, action of cortisol at MR and GR in the heart would also increase. Our present study demonstrates that the effect of cortisol is blocked by antagonists of the MR and/or GR, suggesting a role of intracardiac corticosteroid receptors. This is consistent with the ability of cortisol to alter myocyte growth in cultured myocytes. We also hypothesized that blockade of MR would have a greater effect in inhibiting the effect of cortisol than would blockade of GR, because MR has been shown to have greater affinity for cortisol than GR (Reul \& DeKloet 1985, Richards et al. 2003). Indeed, this is what we observed in the present study: in the cortisol group, there was a $14 \%$ increase in heart weight relative to body weight when compared with the control group; this enlargement was completely blocked when MR antagonist was administered to the heart, whereas there was only $44 \%$ blockade of the increase in weight after administration of the GR antagonist. Similarly, in the cortisol group, LV, RV, and septum thicknesses were $\sim 20 \%$ thicker than control fetuses and the MR antagonist produced a 95, 149, and $114 \%$ reduction of this increase in thickness of the $\mathrm{LV}, \mathrm{RV}$, and

Table 2 Fetal blood gas and packed cell volume. Data are expressed as mean \pm S.E.M

\begin{tabular}{|c|c|c|c|c|}
\hline & Fetal PO2 $(\mathrm{mmHg})$ & Fetal PCO2 $(\mathrm{mmHg})$ & Fetal pH & red blood cells) \\
\hline Control & $21 \cdot 7 \pm 1 \cdot 0$ & $56 \pm 1$ & $7 \cdot 34 \pm 0 \cdot 01$ & $0 \cdot 313 \pm 0 \cdot 007$ \\
\hline Cortisol & $21 \cdot 5 \pm 1 \cdot 0$ & $53 \pm 2$ & $7 \cdot 35 \pm 0.01$ & $0 \cdot 326 \pm 0 \cdot 013$ \\
\hline Cortisol + MRa & $20 \cdot 7 \pm 1 \cdot 1$ & $54 \pm 2$ & $7 \cdot 30 \pm 0.03$ & $0 \cdot 348 \pm 0 \cdot 007$ \\
\hline Cortisol + GRa & $21 \cdot 9 \pm 0 \cdot 4$ & $55 \pm 1$ & $7 \cdot 32 \pm 0 \cdot 02$ & $0.325 \pm 0.009$ \\
\hline
\end{tabular}

Fetal packed cell volume (fractional volume of

Fetal PO2 $(\mathrm{mmHg})$

$20 \cdot 7 \pm 1 \cdot 1$

$21 \cdot 9 \pm 0 \cdot 4$ www.endocrinology-journals.org 
Table 3 Fetal arterial pressure and fetal heart rate on day 10. Data are expressed as mean \pm S.E.M

\begin{tabular}{|c|c|c|}
\hline & $\begin{array}{l}\text { Fetal arterial } \\
\quad \text { pressure }(\mathrm{mmHg})\end{array}$ & $\begin{array}{l}\text { Fetal heart rate } \\
\quad \text { (beats per } \mathrm{min} \text { ) }\end{array}$ \\
\hline Control & $47 \cdot 5 \pm 2 \cdot 7$ & $170 \pm 6$ \\
\hline Cortisol & $46 \cdot 9 \pm 2 \cdot 7$ & $168 \pm 11$ \\
\hline Cortisol + MRa & $43 \cdot 0 . \pm 0 \cdot 9$ & $172 \pm 4$ \\
\hline Cortisol + GRa & $46 \cdot 0 \pm 1 \cdot 0$ & $164 \pm 7$ \\
\hline
\end{tabular}

septum respectively, whereas the GR antagonist group produced 63,110 , and $65 \%$ reductions of thickness. Overall, GR blockade was approximately half as effective as MR blockade in inhibiting the increase in heart weight or wall thickness.

The relative differences in effectiveness of MR and GR blockade are consistent with the expected relative binding of fetal cortisol at these receptors. The MRs are higher affinity receptors with greater occupancy at low cortisol concentrations (Reul \& DeKloet 1985), and therefore a greater effect would be expected after blockade of these receptors. Based on the expected free fraction of cortisol in the fetuses, we calculate that the free cortisol concentrations would be $\sim 0.8 \mathrm{nM}$ in the control fetuses and $1.9 \mathrm{nM}$ in the fetuses of the cortisol-infused ewes. Based on previous studies of cortisol binding at ovine MR and GR (Richards et al. 2003), we would predict that these free concentrations would result in $\sim 65 \%$ occupancy of MR and 35\% occupancy of GR in the control fetuses, and $85 \%$ occupancy of MR and $60 \%$
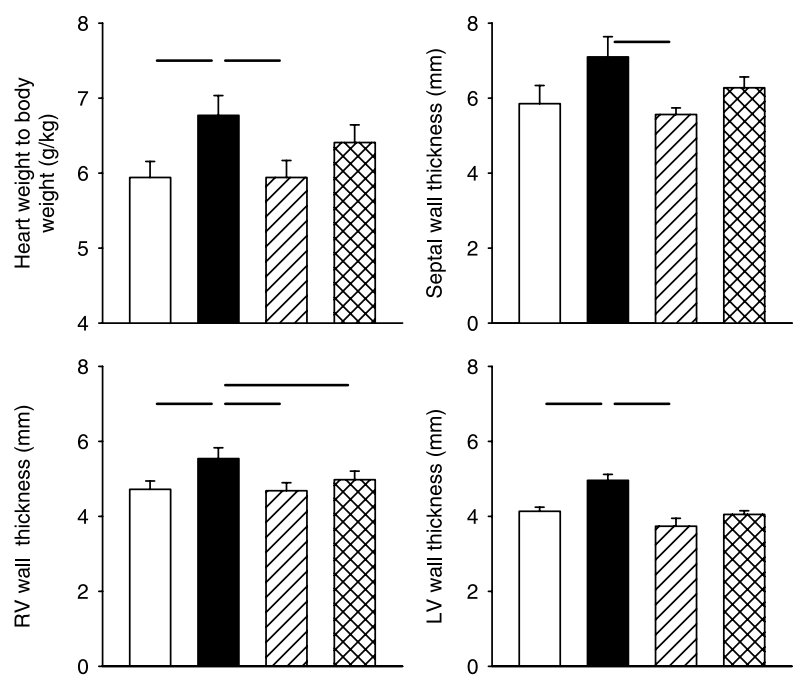

Figure 2 Mean fetal heart measurements from control (open bars), cortisol (solid bars), cortisol + MRa (diagonal striped bars), and cortisol + GRa (cross-hatched bars) groups taken at the time of killing: heart to body mass ratio (upper left), left ventricular (LV) free wall thickness (lower right), septal wall thickness (upper right), and right ventricular (RV) free wall thickness (lower left). Data are expressed as mean \pm s.E.M. Horizontal lines between groups indicate that differences are statistically significant, $P<0 \cdot 05$.
Table 4 Collagen content determined by picrosirius red staining (fraction of total area) in left ventricle (LV), right ventricle (RV), and septum. Data are expressed as mean \pm S.E.M

\begin{tabular}{|c|c|c|c|}
\hline & LV & RV & Septum \\
\hline Control & $0.037 \pm 0.005$ & $0.039 \pm 0.004$ & $0.037 \pm 0.006$ \\
\hline Cortisol & $0.050 \pm 0.009$ & $0.057 \pm 0.009$ & $0.052 \pm 0.010$ \\
\hline Cortisol + MRa & $0 \cdot 049 \pm 0 \cdot 011$ & $0.037 \pm 0.007$ & $0.043 \pm 0.008$ \\
\hline Cortisol + GRa & $0 \cdot 060+0 \cdot 012$ & $0 \cdot 058+0 \cdot 013$ & $0 \cdot 059 \pm 0 \cdot 013$ \\
\hline
\end{tabular}

occupancy of GR in the cortisol-infused fetuses. Thus, these levels would be expected to exert more effects via MR than via GR activation if both act at GRE to induce genes responsible for cardiac growth.

\section{Role of MR in hypertrophy in the adult heart}

In adult rats, the $\mathrm{MR}$ is thought to induce cardiac hypertrophy and fibrosis occurring in response to ischemia; systemic administration of MR blockers have been shown to reduce markers of inflammation and fibrosis in hearts of adult rats (Brilla et al. 1993, Sun et al. 2002, Fraccarollo et al. 2005). It has been established that in adult humans with severe heart failure, there is a reduction in the severity of cardiac hypertrophy and an increase in survival rate after treatment with the MR antagonists, eplenerone or spironolactone (Pitt et al. 1999, 2001). The effect of MR blockers on survival rate appears to be the result of a decrease in cardiac fibrosis (Fraccarollo et al. 2004); increases in interstitial collagen content are a feature of adult cardiac hypertrophy (Pearlman et al. 1981), particularly in the case of hypertension or myocardial infarction (Young et al. 2007). The mechanism for the in vivo effect of MR in contributing to inflammation and subsequent fibrosis is not clear. It has been suggested that the effect is through a non-genomic action, and that the effect in ischemic tissue is predominately on vascular cells expressing MR, rather than on fibroblasts or on myocytes (Mihailidou \& Funder 2005, Young et al. 2007). It is generally assumed that the protective effect of the MR antagonist results from blocking the action of aldosterone at MR. It has been suggested, however, that many heart failure patients without elevated plasma aldosterone levels still benefit from MR blockade, indicating that aldosterone may not be the only relevant MR ligand (Young et al. 2007). Since plasma cortisol concentrations are much higher than aldosterone, and since there is not a significant amount of $11 \beta-H S D 2$ expressed within the heart, it is reasonable to propose that cortisol may be playing a role in the fibrosis that is observed in heart failure patients.

In our studies the effects of cortisol do not appear to involve increase in fibrosis, as there was no increase in collagen content with maternal cortisol infusion, nor were there any effects of either MR or GR blockade. This suggests that the mechanism of the enlargement of the fetal heart in the present study may be fundamentally different from what is observed in 

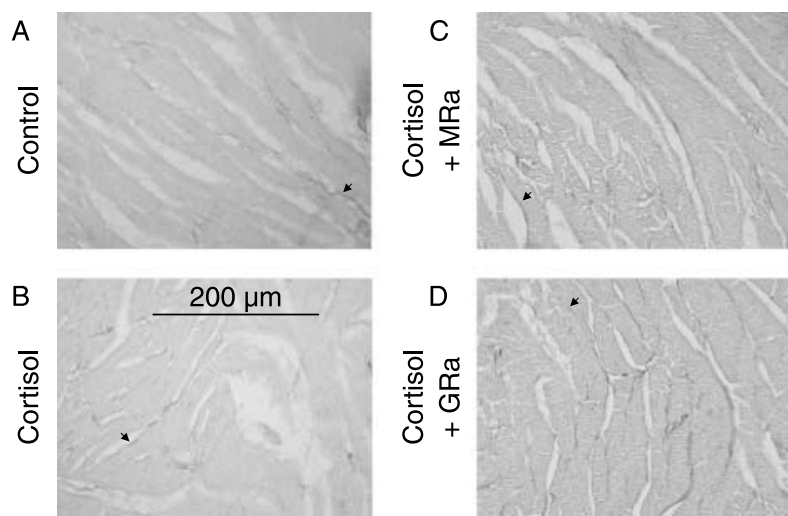

Figure 3 Representative pictures showing picrosirius red staining of collagen in left ventricular wall of fetal hearts from (A) control, (B) cortisol, (C) cortisol + MRa, and (D) cortisol + GRa groups; all photos at $40 \times$ power. Bar indicates $200 \mu \mathrm{m}$. Arrows indicate the dark staining corresponding to positive Sirius red staining.

adult rat models or human pathology, in which ischemia is a contributing component.

\section{Mechanisms of enlargement of the fetal heart}

Due to the unique ability of the fetal heart to grow through both hyperplasia and hypertrophy, either mechanism could account for the cortisol-induced increases in fetal heart weight and wall thickness in our model. In early gestation, cardiac growth is mostly a result of the production of new myocytes originating through cell division and proliferation (Smolich 1995). After $\sim 115$ days of gestation in sheep, however, cardiac growth results primarily from increases in myocyte size (Jonker et al. 2007). Myocytes lose their ability to divide and proliferate shortly after birth in an event in which there is nuclear division without subsequent cell division (Oparil et al. 1984). In fetal sheep, the number of terminally differentiated or binucleate myocytes increases from $\sim 115$ days of gestation through term, and heart growth during this period is due to increases in both myocyte size and myocyte proliferation (Jonker et al. 2007). Theoretically, cortisol could be stimulating growth through either hypertrophy or hyperplasia, or possibly even both.

Rudolph et al. (1999) showed that cortisol $(1 \cdot 2 \mu \mathrm{g} / \mathrm{min})$ infusion for 72-80 h directly into the left coronary artery of the ovine fetus (124-131 days) decreased LV DNA content. This was interpreted as cortisol-induced inhibition of myocyte proliferation in preparation for life after birth. The fetal blood pressures from that study were not reported. In a study by Lumbers et al. (2005) high dose infusion of cortisol $(72 \cdot 1 \mathrm{mg} / \mathrm{d}$ for $\sim 60 \mathrm{~h}$ ) increased LV myocyte size and increase cardiac angiotensinogen mRNA, suggesting an induction of hypertrophy. However, there was also a significant increase in blood pressure in these fetuses, suggesting that the cardiac hypertrophy may have resulted from elevated blood pressure.

Conversely, maternal dexamethasone administration (48 $\mu \mathrm{g} / \mathrm{d}$ from E17) increased relative heart weight and increased myocyte proliferation in the fetal and newborn rat heart (Torres et al. 1997). In agreement with this, Giraud et al. (2006) showed that subpressor doses of cortisol $(0.5 \mu \mathrm{g} / \mathrm{kg}$ per min for 7 days) infused directly into the circumflex coronary artery of the fetus led to an increase in $\mathrm{Ki}-67$ stained myocytes in both the left and RVs; as Ki-67 is expressed only in cells in the proliferative phase, this suggested that cortisol stimulated proliferation in these hearts. Hearts infused with cortisol weighed more than control hearts in this study, but there were no changes in myocyte size or percent binucleation. Interestingly, there were also no differences in aortic, right atrial, systolic, and diastolic pressures between the groups. These studies suggest that elevated fetal cortisol concentrations directly stimulate cardiomyocyte proliferation in the late-term fetus.

The present study does not provide direct evidence for cardiomyocyte proliferation as a means of cardiac enlargement in response to cortisol. It is important to note that in this study a subpressor dose of cortisol was used, as in the study by Giraud et al. (2006). We did not observe an increase in arterial pressure in response to the moderately elevated cortisol levels indicating that the fetal hearts in this study were not subjected to chronically increased systolic load, a possible trigger to myocyte hypertrophy seen in some other studies. Although in the present study blood pressure was only measured at 10 days of cortisol infusion, in our previous study (Jensen et al. 2005) fetal arterial blood pressure was not elevated at either 5 or 10 days of maternal cortisol infusion. The doses of cortisol administered in our study resulted in relatively small increases in fetal cortisol, well below those that have been shown to increase fetal blood pressure in other studies (Wood et al. 1987, Tangalakis et al. 1992, Unno et al. 1999). Furthermore, in this study we observed no evidence within the fetal heart in support of interstitial collagen deposition, a symptom of cardiac hypertrophy in response to hypertension within the adult human heart (Diez 2007).

\section{Conclusions}

The data suggest that the enlargement of the fetal heart in response to a modest and chronic rise in maternal cortisol levels is mediated by MRs and, to a lesser extent, GRs within the fetal heart. Intrapericardial infusion of an MR antagonist completely prevented the increase in wall thickness and heart weight. The GR blockade was less effective, although GR blockade prevented the increase in RV free wall thickness, and tended to attenuate the increase in LV free wall thickness and whole heart weight. The cortisol-induced enlargement is not accompanied by an increase in interstitial collagen deposition within the fetal heart. This indicates the possibility of a different mechanism for the enlargement observed in the fetal heart than that observed in adult cardiac hypertrophy and fibrosis. 


\section{Declaration of Interest}

The authors declare that there is no conflict of interest that would prejudice the impartiality of this scientific work.

\section{Funding}

This work was supported by NIH grant DK62080 to M K-W and American Heart Association Florida-Puerto Rico Affiliate pre-doctoral fellowship grant 0615236B to S A R.

\section{Acknowledgements}

We acknowledge the technical assistance of Jarret McCartney for assistance in the postoperative care of the sheep and for assistance with necropsies. We also acknowledge the assistance of Kavi Patel and Sasha Gidwani for analysis of the collagen content.

\section{References}

Bell ME, Wood CE \& Keller-Wood M 1991 Influence of reproductive state on pituitary-adrenal activity in the ewe. Domestic Animal Endocrinology 8 245-254.

Brilla CG, Matsubara LS \& Weber KT 1993 Antifibrotic effects of spironolactone in preventing myocardial fibrosis in systemic arterial hypertension. American Journal of Cardiology 71 12A-16A.

Diez J 2007 Mechanisms of cardiac fibrosis in hypertension. Journal of Clinical Hypertension 9 546-550.

Fejes-Toth G, Pearce D \& Naray-Fejes-Toth A 1998 Subcellular localization of mineralocorticoid receptors in living cells: effects of receptor agonists and antagonists. PNAS 95 2973-2978.

Fraccarollo D, Galuppo P, Hildemann S, Christ M, Ertl G \& Bauersachs J 2003 Additive improvement of left ventricular remodeling and neurohormonal activation by aldosterone receptor blockade with eplerenone and ACE inhibition in rats with myocardial infarction. Journal of the American College of Cardiology 42 1666-1673.

Fraccarollo D, Galuppo P \& Bauersachs J 2004 Mineralocorticoid receptor antagonism and cardiac remodeling in ischemic heart failure. Current Medicinal Chemistry - Cardiovascular and Hematological Agents 2 287-294.

Fraccarollo D, Galuppo P, Schmidt I, Ertl G \& Bauersachs J 2005 Additive amelioration of left ventricular remodeling and molecular alterations by combined aldosterone and angiotensin receptor blockade after myocardial infarction. Cardiovascular Research 67 97-105.

Giraud GD, Louey S, Jonker S, Schultz J \& Thornburg KL 2006 Cortisol stimulates cell cycle activity in the cardiomyocyte of the sheep fetus. Endocrinology 147 3643-3649.

Gomez-Sanchez CE, de Rodriguez AF, Romero DG, Estess J, Warden MP, Gomez-Sanchez MT \& Gomez-Sanchez EP 2006 Development of a panel of monoclonal antibodies against the mineralocorticoid receptor. Endocrinology 147 1343-1348.

Jensen E, Wood C \& Keller-Wood M 2002a The normal increase in adrenal secretion during pregnancy contributes to maternal volume expansion and fetal homeostasis. Journal of the Society for Gynecologic Investigation 9 362-371.

Jensen EC, Gallaher BW, Breier BH \& Harding JE $2002 b$ The effect of a chronic maternal cortisol infusion on the late-gestation fetal sheep. Journal of Endocrinology 174 27-36.

Jensen E, Wood CE \& Keller-Wood M 2005 Chronic alterations in ovine maternal corticosteroid levels influence uterine blood flow and placental and fetal growth. American Journal of Physiology. Regulatory, Integrative and Comparative Physiology 288 R54-R61.

Jewell CM, Webster JC, Burnstein KL, Sar M, Bodwell JE \& Cidlowski JA 1995 Immunocytochemical analysis of hormone mediated nuclear translocation of wild type and mutant glucocorticoid receptors. Journal of Steroid Biochemistry and Molecular Biology 55 135-146.
Jonker SS, Zhang L, Louey S, Giraud GD, Thornburg KL \& Faber JJ 2007 Myocyte enlargement, differentiation, and proliferation kinetics in the fetal sheep heart. Journal of Applied Physiology 102 1130-1142.

Kliche K, Kuhn M, Hillebrand U, Ludwig Y, Stock C \& Oberleithner H 2006 Direct aldosterone action on mouse cardiomyocytes detected with atomic force microscopy. Cellular Physiology and Biochemistry 18 265274.

Krozowski Z, Li KX, Koyama K, Smith RE, Obeyesekere VR, Stein-Oakley A, Sasano H, Coulter C, Cole T \& Sheppard KE 1999 The type I and type II 11beta-hydroxysteroid dehydrogenase enzymes. Journal of Steroid Biochemistry and Molecular Biology 69 391-401.

Lister K, Autelitano DJ, Jenkins A, Hannan RD \& Sheppard KE 2006 Cross talk between corticosteroids and alpha-adrenergic signalling augments cardiomyocyte hypertrophy: a possible role for SGK1. Cardiovascular Research 70 555-565.

Lombes M, Binart N, Delahaye F, Baulieu EE \& Rafestin-Oblin ME 1994 Differential intracellular localization of human mineralocorticosteroid receptor on binding of agonists and antagonists. Biochemical Journal $\mathbf{3 0 2}$ 191-197.

Lumbers ER, Boyce AC, Joulianos G, Kumarasamy V, Barner E, Segar JL \& Burrell JH 2005 Effects of cortisol on cardiac myocytes and on expression of cardiac genes in fetal sheep. American Journal of Physiology. Regulatory, Integrative and Comparative Physiology 288 R567-R574.

Mihailidou AS \& Funder JW 2005 Nongenomic effects of mineralocorticoid receptor activation in the cardiovascular system. Steroids 70 347-351.

Okoshi MP, Yan X, Okoshi K, Nakayama M, Schuldt AJ, O'Connell TD, Simpson PC \& Lorell BH 2004 Aldosterone directly stimulates cardiac myocyte hypertrophy. Journal of Cardiac Failure 10 511-518.

Oparil S, Bishop SP \& Clubb FJ Jr 1984 Myocardial cell hypertrophy or hyperplasia. Hypertension 6 III38-III43.

Pearlman ES, Weber KT \& Janicki JS 1981 Quantitative histology of the hypertrophied human heart. Federation Proceedings 40 2042-2047.

Pitt B, Zannad F, Remme WJ, Cody R, Castaigne A, Perez A, Palensky J \& Wittes J 1999 The effect of spironolactone on morbidity and mortality in patients with severe heart failure. Randomized Aldactone Evaluation Study Investigators. New England Journal of Medicine 341 709-717.

Pitt B, Williams G, Remme W, Martinez F, Lopez-Sendon J, Zannad F, Neaton J, Roniker B, Hurley S, Burns D et al. 2001 The EPHESUS trial: eplerenone in patients with heart failure due to systolic dysfunction complicating acute myocardial infarction. Eplerenone Post-AMI Heart Failure Efficacy and Survival Study. Cardiovascular Drugs and Therapy 15 79-87.

Reini SA, Wood CE, Jensen E \& Keller-Wood M 2006 Increased maternal cortisol in late gestation ewes decreases fetal cardiac expression of $11 \beta$ HSD 2 mRNA and the ratio of AT1 to AT2 receptor mRNA. American Journal of Physiology. Regulatory, Integrative and Comparative Physiology 21 R1708-R1716.

Reul JMHM \& DeKloet ER 1985 Two receptor systems for corticosterone in rat brain: microdistribution and differential occupation. Endocrinology 117 2505-2511.

Richards EM, Hua Y \& Keller-Wood M 2003 Pharmacology and physiology of ovine corticosteroid receptors. Neuroendocrinology 77 2-14.

Rudolph AM, Roman C \& Gournay V 1999 Perinatal myocardial DNA and protein changes in the lamb: effect of cortisol in the fetus. Pediatric Research 46 141-146.

Scheuer DA, Bechtold AG, Shank SS \& Akana SF 2004 Glucocorticoids act in the dorsal hindbrain to increase arterial pressure. American Journal of Physiology. Heart and Circulatory Physiology 286 H458-H467.

Smolich JJ 1995 Ultrastructural and functional features of the developing mammalian heart: a brief overview. Reproduction, Fertility, and Development 7 451-461.

Sun Y, Zhang J, Lu L, Chen SS, Quinn MT \& Weber KT 2002 Aldosteroneinduced inflammation in the rat heart: role of oxidative stress. American Journal of Pathology 161 1773-1781.

Tangalakis K, Lumbers ER, Moritz KM, Towstoless MK \& Wintour EM 1992 Effect of cortisol on blood pressure and vascular reactivity in the ovine fetus. Experimental Physiology 77 709-717. 
Torres A, Belser WW III, Umeda PK \& Tucker D 1997 Indicators of delayed maturation of rat heart treated prenatally with dexamethasone. Pediatric Research 42 139-144.

Unno N, Wong CH, Jenkins SL, Wentworth RA, Ding XY, Li C, Robertson SS, Smotherman WP \& Nathanielsz PW 1999 Blood pressure and heart rate in the ovine fetus: ontogenic changes and effects of fetal adrenalectomy. American Journal of Physiology 276 H248-H256.

Winer BJ 1971 Statistical Principles in Experimental Design., New York: McGraw-Hill.

Wood CE 2002 The ovine fetal endocrine reflex responses to haemorrhage are not mediated by cardiac nerves. Journal of Physiology 541 613-622.

Wood CE \& Rudolph AM 1983 Negative feedback regulation of adrenocorticotropin secretion by cortisol. Endocrinology 112 1930-1936.

Wood CE, Cheung CY \& Brace RA 1987 Fetal heart rate, arterial pressure, and blood volume responses to cortisol infusion. American Journal of Physiology 253 R904-R909.
Xiao HD, Fuchs S, Campbell DJ, Lewis W, Dudley SC Jr, Kasi VS, Hoit BD, Keshelava G, Zhao H, Capecchi MR et al. 2004 Mice with cardiacrestricted angiotensin-converting enzyme (ACE) have atrial enlargement, cardiac arrhythmia, and sudden death. American Journal of Pathology 165 1019-1032.

Young MJ, Lam EY \& Rickard AJ 2007 Mineralocorticoid receptor activation and cardiac fibrosis. Clinical Science 112 467-475.

Zar JH 1984 Biostatistical Analysis., Englewood Cliffs, NJ: Prentice-Hall.

Received in final form 22 April 2008

Accepted 20 May 2008

Made available online as an Accepted Preprint 21 May 2008 